\title{
Biogenerics at the crossroads
}

\author{
Carole S Ben-Maimon \& Rob Garnick
}

\section{Two executives - one from a leading generics manufacturer, the other from a leading brand manufacturer-argue the pros and cons of implementing an abbreviated US regulatory framework for the approval of biogeneric products.}

\begin{abstract}
The battle for enhanced savings in prescription medicine is about to take center stage in the arena of regulatory and political debate, and the winners could be US consumers. The stakes are high. Biopharmaceutical products currently have annual sales exceeding $\$ 30$ billion and several of these products, which were developed during the 1980s, have lost, or will soon lose, patent protection. Sources estimate that within the next five years, biopharmaceutical products with annual sales in excess of $\$ 10$ billion will lose patent protection. These products would be eligible for competition from generic biopharmaceuticals if an approval process existed. Below, executives from a leading generics manufacturer and a leading brand biologic manufacturer present arguments for and against the introduction of such a mechanism for biogeneric approval in the United States.
\end{abstract}

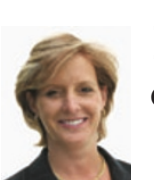

\section{POINT: Why biogenerics make sense}

\author{
Carole S Ben-Maimon
}

If the US Congress and the US Food and Drug Administration (FDA) act based upon science, and not upon the rhetoric of special interests, they will create a pathway for the approval of biogenerics. This will allow consumers who spend tens of thousands of dollars each year on biopharmaceutical products to be rewarded with the savings that result from the introduction of generic competition for expensive but essential therapies.

Biogenerics are approvable today. The fact is that no scientific, clinical or manufacturing hurdles exist today to prevent the introduction of affordable generic biopharmaceuticals. The question regarding the introduction of generic biopharmaceuticals in the United States is not 'if', but 'when' and which ones first.

The principal obstacle involves establishing an abbreviated approval process that will result in the ability of the FDA to evaluate and approve generic biopharmaceuticals that can

Carole S. Ben-Maimon is President and CEO of Barr Pharmaceuticals, 400 Chesnut Ridge Road, Woodcliff Lake, NJ 07677, USA.

e-mail:amanno@barrlabs.com be substituted for the brand-name product. Currently, manufacturers of generic drugs could bring their own versions of biopharmaceuticals to the marketplace, but the process would require a full development program and the approval obtained would result in a 'new' biopharmaceutical product-one that would need to be marketed and promoted to individual doctors-not one that could be directly substituted for the brand-name version at the pharmacy, as traditional generic pharmaceutical drugs are today.

Whereas Congress and the FDA have been moving at a glacial pace on the issue of generic biologics, the European Medicines Agency (EMEA) passed guidelines in November 2005 that streamline the process for the development and approval of generic biopharmaceuticals. According to a report in the Financial Times, "Observers are speculating that the EMEA will grant approval for the first wave of biosimilars as early as next year... While Europe may be racing down the road to biosimilars, the US has so far dragged its heels. The US is not expected to come to any decision before 2007 about shortening the approval process for biosimilars, which will leave Europe in the unusual position of leading the US on a scientific issue."

Hollow counterarguments. Opponents attempting to delay the establishment of a pathway for the approval of generic biopharmaceuticals have raised a number of 'issues,' which they claim demonstrate that approval of generic biopharmaceuticals is not possible. Their argument revolves around the premise that biopharmaceutical drugs are so complex that they cannot be characterized. This is, in part, based on the mistaken assumption that manufacturers of generics do not have the technological expertise or scientific, medical or clinical capabilities to safely develop biogeneric drugs. Although it is clear that some biopharmaceutical products might be more complex, the vast majority can be fully characterized. This is a continum no different from that with drug products. And advances in scientific, clinical and analytical methods will ultimately enable the characterization of all drugs.

Safety issues. Raw materials are available today for many biogeneric products including insulin, granulocyte-macrophage colonystimulating factor, erythropoietin, interferons and others. And in many countries around the world, competitive biopharmaceutical drugs are already approved and marketed without unintended effects-clearly demonstrating that safety is not an issue. With appropriate regulatory oversight, safety will not be an issue in the United States either.

I question the claim that there is 'magic' to the process of manufacturing biopharmaceutical drugs and that large development programs costing hundreds of millions of dollars are necessary to prove the safety and effectiveness of a generic version. This is a double standard when a brand biopharmaceutical company seeks changes in processes supporting the manufacture of their products or seeks to change the manufacturing loca- 
tion of a product. In such cases, the FDA does not require the brand company to conduct full-scale clinical trials to confirm safety and efficacy.

A robust and reproducible process that yields a final product that consistently matches the desired composition of the reference product should be acceptable for the manufacturing of safe, effective and equivalent generic biopharmaceuticals.

With more than two decades of experience with many brand biopharmaceuticals, our knowledge of the causes of immunogenicity for specific products has increased significantly. Analytical testing of biopharmaceuticals can be used to minimize the potential for changes in the immunogenicity of a specific product.

The time has come. The substantial savings resulting from the entrance into the market of generic biopharmaceuticals will only be fully realized when a process is formalized that results in the approval of a generic product that can be directly substituted for the brandname version. For this reason, the US Congress and FDA must create a regulatory process that will help make biopharmaceuticals available and affordable for all by enabling the timely, efficient and cost-effective approval of generic versions of biopharmaceuticals.

The principles supporting the creation of an abbreviated approval pathway are clear. It must be based on sound scientific rationale that does not require unnecessary trials that have previously been conducted by the innovator. Clinical study requirements must be directly related to the complexity of the molecule. For those biopharmaceuticals that are less complex, characterization must be the cornerstone for approval. For moderately complex biopharmaceuticals, clinical studies must be limited, and trials should only be as large as necessary to scientifically demonstrate comparability of the generic to the brand-name product.

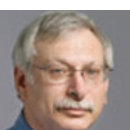

\section{COUNTERPOINT: Why biogenerics are a strawman}

\author{
Rob Garnick
}

US state and federal lawmakers are under the dual public policy pressures of both ensuring patient access to cheaper drugs and providing incentives for research companies to continue to innovate and invest resources in the search for breakthrough medical therapies. In trying to balance these goals, they must also be mindful that above all, individuals should have access to medicines that are safe and effective, not just inexpensive.

Several policy proposals have been suggested that seek to make drugs and drug development less expensive, possibly at the expense of safety and efficacy. The debate over 'follow-on' or 'generic' biologics is one suggested solution that would not necessarily result in less expensive drugs, and could put public safety at greater risk.

Beware of oversimplification. For some, the solution is simple: develop an approval pathway for generic or follow-on versions of biopharmaceuticals to obtain savings like those achieved with generic drugs. Although this solution may appear obvious and straightforward; in fact, it is far more

Rob Garnick, is Senior Vice President,

Regulatory, Quality and Compliance,

Genentech, 1 DNA Way, South San Francisco,

CA 94080, USA.

e-mail: snyder.robin@gene.com complicated than what some may think. The very nature of biopharmaceuticals raises serious scientific and legal questions regarding the validity and advisability of moving forward with an approval pathway for follow-on biotech products. The FDA should not proceed with developing such a pathway until these crucial questions are answered.

Biotech-derived products are made through intricate manufacturing processes that depend on living organisms (i.e., highly characterized cell lines). Unlike traditional pharmaceuticals, good scientific practice does not allow the direct comparison of one biotech product to another. This is because complex operational and proprietary details of the biotech manufacturing process are central to, and define the identity and unique structural characteristics of, each biotechderived product.

Accordingly, such processes are integral in determining the safety and effectiveness of those products. Because each biopharmaceutical manufacturing process is necessarily unique, and because small changes to any such process can have profound consequences on the end product, the FDA cannot rely on the analytical data generated from one biotech product to support approval of a product manufactured using another cell line and with different proprietary processes.
Small versus large molecules. Traditional small-molecule pharmaceuticals, on the other hand, are not made in living cells, but instead are made using highly reproducible processes involving chemical analysis. As such, small-molecule drugs can be defined completely by their atomic structure rather than by their manufacturing processes. In addition, a small-molecule drug can be shown, through chemical analysis, to be the same as another, fully analyzed and approved small-molecule product. As such, the safety and efficacy data generated for a small molecule by the innovator is relevant and applicable to the generic product. Because the products can be shown to be the same, the FDA can approve the generic product, without significant additional testing requirements and with confidence that the generic product is safe and effective.

Given this fundamental difference between biopharmaceuticals and more traditional, small-molecule drugs, the current paradigm for reviewing and approving generics is simply not applicable. As safety and efficacy data are not transferable between biopharmaceutical products, there can be no assurance that the follow-on product is safe or effective, as in the smallmolecule example.

Why we're not ready. The fact that biomanufacturing processes profoundly affect biotech products presents several problems to any abbreviated process for producing generic biopharmaceuticals. First, the proposed generic or follow-on product would be manufactured using an entirely different cell line, plasmid and process, as these materials and information belong to the biotech company and are closely guarded proprietary materials and trade secrets.

Second, to make effective comparisons between products would require that the FDA rely on a company's trade-secret manufacturing data. Were the FDA allowed to rely on a company's confidential and proprietary manufacturing data to the direct benefit of a competitor, there would be little incentive for a brand manufacturer to continue to invest the time and resources necessary to bringing breakthrough products to market.

As we continue to debate and consider approaches to providing patents with less expensive yet more effective and safe drugs, we must not rush toward solutions that appear expedient, yet are unsound scientifically and economically, and that could provide disincentives for innovators to continue to invest in discovering and developing new cures. 


\section{Erratum: Biotech entrepreneur, educate thyself!}

\section{Emily Waltz}

Bioentrepreneur, published online 15 December 2005, corrected 27 March 2006; doi:10.1038/bioent896

In the online version of this article (paragraph 4), Chad Walton is incorrectly said to have graduated from a dual-degree program. The text should read, “...Chad Walton, who received a master of biomedical technology (MBT) from the University of Calgary....” The program with the eight students he is referring to is the MBT program, not the dual-degree program. The MBT program does, however, have a business component. The error has been corrected in the HTML version of the article.

\section{Erratum: European GMO labeling thresholds impractical and unscientific} Florian Weighardt

Nat. Biotechnol. 24, 23-25 (2006), corrected 27 March 2006

In the print version of this article and the version published online, several inaccuracies were introduced into the text through editorial modifications. On page 23, column 2, rows 3-4, the text: "It is now more than three years since the legislation was first introduced...." should have read "...more than five years...." On page 24, column 1, rows 35-37: "Currently, the method of choice for transgenic sequence detection is real-time PCR." should have read “...for transgenic sequence quantification...." Page 24, column 1, rows 51-55: "CRMs are prepared from certified seeds of the GM inbred line and of the non-GM parental variety used for the transformation are homogenized and grinded to powder." should have read, "For the GM inbred line and the non-GM parental variety used for the transformation, CRMs are prepared from certified seeds, homogenized and grinded to powder." On page 25, column 1, rows 22-25: "The EU legislators continue to fudge; the current regulation 1829/2003 (ref. 4) uses the same imprecise 1\% threshold as its predecessor $49 / 2000$ (ref. 2). And the EC's most recent recommendation $(2004 / 787 / 2000)^{17}$ only partly...." should have read “....uses the same imprecise threshold definition as its predecessor 49/2000, which introduced a 1\% threshold ${ }^{2}$. And the EC's most recent recommendation (2004/787/ EC) only partly..." The errors have been corrected in the PDF version of the article.

\section{Erratum: Biotech entrepreneur, educate thyself!}

Emily Waltz

Nat. Biotechnol. 24, 131-132 (2006), corrected 27 March 2006

In the print version of this article (paragraph 4 and picture caption), Chad Walton is incorrectly said to have graduated from a dual-degree program. The text should read, “...Chad Walton, who received a master of biomedical technology (MBT) from the University of Calgary....” The caption should read, "Entrepreneur and MBT graduate Chad Walton: just do it." The program with the eight students he is referring to is the MBT program, not the dual-degree program. The MBT program does, however, have a business component. The error has been corrected in the PDF version of the article.

\section{Erratum: Biogenerics at the crossroads}

\section{Carole S Ben-Maimon \& Rob Garnick}

Nat. Biotechnol. 24, 268 (2006), corrected 27 March 2006

In the print version of this article and the version originally published online, Carole Ben-Maimon was incorrectly listed as president and CEO of Barr Pharmaceuticals. She is, in fact, president and COO of Duramed Research, a subsidiary of Barr Pharmaceuticals. Her email address was also incorrect; her correct email address is cbenmaimon@barrlabs.com. The error has been corrected in the PDF version of the article. 


\title{
Biogenerics at the crossroads
}

\author{
Carole S Ben-Maimon \& Rob Garnick
}

\section{Two executives - one from a leading generics manufacturer, the other from a leading brand manufacturer-argue the pros and cons of implementing an abbreviated US regulatory framework for the approval of biogeneric products.}

\begin{abstract}
The battle for enhanced savings in prescription medicine is about to take center stage in the arena of regulatory and political debate, and the winners could be US consumers. The stakes are high. Biopharmaceutical products currently have annual sales exceeding $\$ 30$ billion and several of these products, which were developed during the 1980s, have lost, or will soon lose, patent protection. Sources estimate that within the next five years, biopharmaceutical products with annual sales in excess of $\$ 10$ billion will lose patent protection. These products would be eligible for competition from generic biopharmaceuticals if an approval process existed. Below, executives from a leading generics manufacturer and a leading brand biologic manufacturer present arguments for and against the introduction of such a mechanism for biogeneric approval in the United States.
\end{abstract}

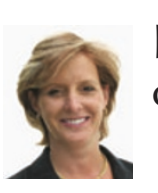

\section{POINT: Why biogenerics make sense}

Carole S Ben-Maimon

If the US Congress and the US Food and Drug Administration (FDA) act based upon science, and not upon the rhetoric of special interests, they will create a pathway for the approval of biogenerics. This will allow consumers who spend tens of thousands of dollars each year on biopharmaceutical products to be rewarded with the savings that result from the introduction of generic competition for expensive but essential therapies.

Biogenerics are approvable today. The fact is that no scientific, clinical or manufacturing hurdles exist today to prevent the introduction of affordable generic biopharmaceuticals. The question regarding the introduction of generic biopharmaceuticals in the United States is not 'if', but 'when' and which ones first.

Carole S. Ben-Maimon is president and COO of Duramed Research, a subsidiary of Barr Pharmaceuticals, 400 Chesnut Ridge Road, Woodcliff Lake, NJ 07677, USA. e-mail: cbenmaimon@barrlabs.com

Published online 7 March 2006; corrected after print 27 March 2006
The principal obstacle involves establishing an abbreviated approval process that will result in the ability of the FDA to evaluate and approve generic biopharmaceuticals that can be substituted for the brand-name product. Currently, manufacturers of generic drugs could bring their own versions of biopharmaceuticals to the marketplace, but the process would require a full development program and the approval obtained would result in a 'new' biopharmaceutical product—one that would need to be marketed and promoted to individual doctors - not one that could be directly substituted for the brand-name version at the pharmacy, as traditional generic pharmaceutical drugs are today.

Whereas Congress and the FDA have been moving at a glacial pace on the issue of generic biologics, the European Medicines Agency (EMEA) passed guidelines in November 2005 that streamline the process for the development and approval of generic biopharmaceuticals. According to a report in the Financial Times, "Observers are speculating that the EMEA will grant approval for the first wave of biosimilars as early as next year... While Europe may be racing down the road to biosimilars, the US has so far dragged its heels. The US is not expected to come to any decision before 2007 about shortening the approval process for biosimilars, which will leave Europe in the unusual position of leading the US on a scientific issue."

Hollow counterarguments. Opponents attempting to delay the establishment of a pathway for the approval of generic biopharmaceuticals have raised a number of 'issues,' which they claim demonstrate that approval of generic biopharmaceuticals is not possible. Their argument revolves around the premise that biopharmaceutical drugs are so complex that they cannot be characterized. This is, in part, based on the mistaken assumption that manufacturers of generics do not have the technological expertise or scientific, medical or clinical capabilities to safely develop biogeneric drugs. Although it is clear that some biopharmaceutical products might be more complex, the vast majority can be fully characterized. This is a continum no different from that with drug products. And advances in scientific, clinical and analytical methods will ultimately enable the characterization of all drugs.

Safety issues. Raw materials are available today for many biogeneric products including insulin, granulocyte-macrophage colonystimulating factor, erythropoietin, interferons and others. And in many countries around the world, competitive biopharmaceutical drugs are already approved and marketed without unintended effects-clearly demonstrating that safety is not an issue. With appropriate regulatory oversight, safety will not be an issue in the United States either.

I question the claim that there is 'magic' to the process of manufacturing biopharmaceutical drugs and that large development programs costing hundreds of millions of dollars are necessary to prove the safety and effectiveness of a generic version. This is a double standard when a brand biopharmaceutical company seeks changes in processes supporting the manufacture of their products or seeks to change the 
manufacturing location of a product. In such cases, the FDA does not require the brand company to conduct full-scale clinical trials to confirm safety and efficacy.

A robust and reproducible process that yields a final product that consistently matches the desired composition of the reference product should be acceptable for the manufacturing of safe, effective and equivalent generic biopharmaceuticals.

With more than two decades of experience with many brand biopharmaceuticals, our knowledge of the causes of immunogenicity for specific products has increased significantly. Analytical testing of biopharmaceuticals can be used to minimize the potential for changes in the immunogenicity of a specific product.

The time has come. The substantial savings resulting from the entrance into the market of generic biopharmaceuticals will only be fully realized when a process is formalized that results in the approval of a generic product that can be directly substituted for the brandname version. For this reason, the US Congress and FDA must create a regulatory process that will help make biopharmaceuticals available and affordable for all by enabling the timely, efficient and cost-effective approval of generic versions of biopharmaceuticals.

The principles supporting the creation of an abbreviated approval pathway are clear. It must be based on sound scientific rationale that does not require unnecessary trials that have previously been conducted by the innovator. Clinical study requirements must be directly related to the complexity of the molecule. For those biopharmaceuticals that are less complex, characterization must be the cornerstone for approval. For moderately complex biopharmaceuticals, clinical studies must be limited, and trials should only be as large as necessary to scientifically demonstrate comparability of the generic to the brandname product.

\title{
COUNTERPOINT: Why biogenerics are a strawman
}

\author{
Rob Garnick
}

US state and federal lawmakers are under the dual public policy pressures of both ensuring patient access to cheaper drugs and providing incentives for research companies to continue to innovate and invest resources in the search for breakthrough medical therapies. In trying to balance these goals, they must also be mindful that above all, individuals should have access to medicines that are safe and effective, not just inexpensive.

Several policy proposals have been suggested that seek to make drugs and drug development less expensive, possibly at the expense of safety and efficacy. The debate over 'follow-on' or 'generic' biologics is one suggested solution that would not necessarily result in less expensive drugs, and could put public safety at greater risk.

Beware of oversimplification. For some, the solution is simple: develop an approval pathway for generic or follow-on versions of biopharmaceuticals to obtain savings like those achieved with generic drugs. Although this solution may appear obvious and straightforward; in fact, it is far more

Rob Garnick, is Senior Vice President,

Regulatory, Quality and Compliance,

Genentech, 1 DNA Way, South San Francisco,

CA 94080, USA.

e-mail: snyder.robin@gene.com

complicated than what some may think. The very nature of biopharmaceuticals raises serious scientific and legal questions regarding the validity and advisability of moving forward with an approval pathway for follow-on biotech products. The FDA should not proceed with developing such a pathway until these crucial questions are answered.

Biotech-derived products are made through intricate manufacturing processes that depend on living organisms (i.e., highly characterized cell lines). Unlike traditional pharmaceuticals, good scientific practice does not allow the direct comparison of one biotech product to another. This is because complex operational and proprietary details of the biotech manufacturing process are central to, and define the identity and unique structural characteristics of, each biotechderived product.

Accordingly, such processes are integral in determining the safety and effectiveness of those products. Because each biopharmaceutical manufacturing process is necessarily unique, and because small changes to any such process can have profound consequences on the end product, the FDA cannot rely on the analytical data generated from one biotech product to support approval of a product manufactured using another cell line and with different proprietary processes.
Small versus large molecules. Traditional small-molecule pharmaceuticals, on the other hand, are not made in living cells, but instead are made using highly reproducible processes involving chemical analysis. As such, small-molecule drugs can be defined completely by their atomic structure rather than by their manufacturing processes. In addition, a small-molecule drug can be shown, through chemical analysis, to be the same as another, fully analyzed and approved small-molecule product. As such, the safety and efficacy data generated for a small molecule by the innovator is relevant and applicable to the generic product. Because the products can be shown to be the same, the FDA can approve the generic product, without significant additional testing requirements and with confidence that the generic product is safe and effective.

Given this fundamental difference between biopharmaceuticals and more traditional, small-molecule drugs, the current paradigm for reviewing and approving generics is simply not applicable. As safety and efficacy data are not transferable between biopharmaceutical products, there can be no assurance that the follow-on product is safe or effective, as in the smallmolecule example.

Why we're not ready. The fact that biomanufacturing processes profoundly affect biotech products presents several problems to any abbreviated process for producing generic biopharmaceuticals. First, the proposed generic or follow-on product would be manufactured using an entirely different cell line, plasmid and process, as these materials and information belong to the biotech company and are closely guarded proprietary materials and trade secrets.

Second, to make effective comparisons between products would require that the FDA rely on a company's trade-secret manufacturing data. Were the FDA allowed to rely on a company's confidential and proprietary manufacturing data to the direct benefit of a competitor, there would be little incentive for a brand manufacturer to continue to invest the time and resources necessary to bringing breakthrough products to market.

As we continue to debate and consider approaches to providing patents with less expensive yet more effective and safe drugs, we must not rush toward solutions that appear expedient, yet are unsound scientifically and economically, and that could provide disincentives for innovators to continue to invest in discovering and developing new cures. 